\title{
Average Sum-Rate of Distributed Alamouti Space-Time Scheme in Two-Way Amplify-and-Forward Relay Networks
}

\author{
Trung Q. Duong ${ }^{\dagger}$, Chau Yuen ${ }^{\ddagger}$, Hans-Jürgen Zepernick ${ }^{\dagger}$, and Xianfu Lei ${ }^{\S}$ \\ ${ }^{\dagger}$ Blekinge Institute of Technology, SE-371 79, Karlskrona, Sweden \\ ${ }^{\ddagger}$ Singapore University of Technology and Design, Singapore \\ ${ }^{\S}$ Southwest Jiaotong University, Chengdu 610031, China \\ E-mail: dqt@bth.se,yuenchau@sutd.edu.sg, hjz@bth.se, xflei81@yahoo.com.cn
}

\begin{abstract}
In this paper, we propose a distributed Alamouti space-time code (DASTC) for two-way relay networks employing a single amplify-and-forward (AF) relay. We first derive closedform expressions for the approximated average sum-rate of the proposed DASTC scheme. Our analysis is validated by a comparison against the results of Monte-Carlo simulations. Numerical results verify the effectiveness of our proposed scheme over the conventional DASTC with one-way communication.
\end{abstract}

\section{INTRODUCTION}

Relay networking has been considered as an efficient approach to increase the communication range of wireless systems. However, dual-hop half-duplex relay networks lose half of the throughput compared to the direct communication due to the fact that the relay cannot transmit and receive simultaneously. To overcome this drawback, a two-way (or bi-directional) relay network has been presented in [1], where two nodes, namely $S_{1}$ and $S_{2}$, transmit simultaneously to the relay $\mathrm{R}$ in the first hop, and in the second hop the relay $\mathrm{R}$ forwards its received signals to both terminals $S_{1}$ and $S_{2}$. With this strategy, this loss can be remarkably compensated.

As a result, two-way relay networks have gained great attention in the research community (e.g., see [1]-[5]). In view of the related work of two-way relay networks, the upper and lower bounds for average sum-rate have been investigated in [1]. The performance of two-way amplify-and-forward (AF) relay with network coding has been investigated in [2]. By giving up the strict separation of downlink and uplink signals through either time or frequency division duplex, a two-hop relaying, namely space division duplex relaying, is proposed in [3]. The exact closed-form expressions of error probability, average sum-rate for two-way AF relays have been presented in [4], [5].

Distributed Alamouti space-time code (DASTC) with AF relays originally applied for one-way relay networks [6] has recently extended to two-way systems (see, e.g., [7]-[9]). In particular, a two-way relaying scheme where two sources equipped with two antennas transmit Alamouti code through the help of an AF relay has been proposed in [7], [8]. Upper and lower bounds of average sum-rate have also been derived for this particular bi-directional relaying system. Another system in which the burden of deploying multiple antennas on sources is transferred to the relay has been proposed in [9]. By assuming that the relay is equipped with two antennas and each source is equipped with a single antenna, an upper bound of symbol error probability has been obtained. As can be observed from all the above schemes, either relay or source requires multiple antennas which may be prohibited in practical implementations due to the high demand for low-cost and small-size portable devices.

To alleviate this requirement and make the system realistic, a DASTC scheme which all terminals having only one antenna has been presented for one-way AF relay networks [10], [11]. However, this system still faces loss in spectral efficiency. Hence, in this paper, we propose a DASTC scheme for two-way AF relay networks which significantly recovers this loss. More importantly, unlike the analysis work in [7], [8] where the bounds of average sum-rate have been shown, we have derived an asymptotically tight approximation for the average sum-rate of the proposed two-way scheme. The final expression is given in the form of Fox's H-function which enables us to investigate the performance of the proposed scheme. In addition, we also provide the numerical results to verify the correctness of our analysis.

The rest of this paper is organized as follows. In Section II we introduce the system model of the proposed DASTC for two-way AF relay networks. Then, in Section III, we derive the tight approximation for the average sum-rate of the proposed scheme. Numerical results are shown in Section IV to validate the analysis. Finally, we conclude the paper in Section $\mathrm{V}$

Notation: Throughout the paper, we shall use the following notation. Vector is written as bold lower case letter and matrix is written as bold upper case letter. The superscripts $*$ and $\dagger$ stand for the complex conjugate and transpose conjugate, respectively. $\boldsymbol{I}_{n}$ represents the $n \times n$ identity matrix. $\|\boldsymbol{A}\|_{\mathrm{F}}$ denotes Frobenius norm of the matrix $\boldsymbol{A}$ and $|x|$ indicates the envelope of $x . \mathbb{E}_{x}\{$.$\} is the expectation operator over the ran-$ dom variable $x$. A complex Gaussian distribution with mean $\mu$ and variance $\sigma^{2}$ is denoted by $\mathcal{C N}\left(\mu, \sigma^{2}\right)$. Let us denote $\tilde{\mathcal{N}}_{m}(\boldsymbol{m}, \boldsymbol{\Sigma})$ as a complex Gaussian random vector with mean vector $\boldsymbol{m}$ and covariance matrix $\boldsymbol{\Sigma}$. $\Gamma(a, x)$ is the incomplete gamma function defined as $\Gamma(a, x)=\int_{x}^{\infty} t^{a-1} e^{-t} d t$ and $\mathcal{K}_{n}($.$) is the n$ th-order modified Bessel function of the second 


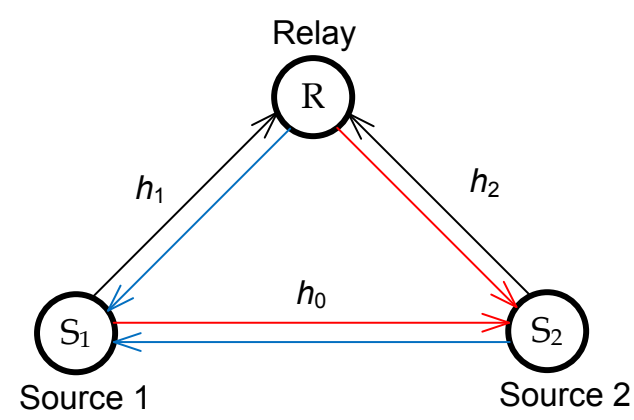

Fig. 1. System model.

kind.

\section{System Model of Distributed Alamouti SPACE-TIME CODES With Two-WAy AF RElaying}

In this section, we introduce the system model of the proposed DASTC scheme with two-way AF relaying. Consider a wireless AF relay network consisting of three terminals as shown in Fig. 1. Two sources, namely $S_{1}$ and $S_{2}$, exchange the information through the assistance of an AF relay R. Each terminal is equipped with a single antenna which operates in half-duplex mode. We also assume that the channel for all links induces quasi-static fading, i.e., the channel remains constant for a block spanning over at least six symbolsintervals and varies independently for every block. Due to the channel reciprocity, we note that channel gains of $A \longrightarrow B$ and $B \longrightarrow A$ links, with $A, B \in\left\{S_{1}, S_{2}, R\right\}$, are identical.

The communications of the proposed two-way AF DASTC scheme occur in three phases. In the first phase, both $S_{1}$ and $\mathrm{S}_{2}$ transmit the first row of Alamouti code [12] to $\mathrm{R}$ with the same transmit power per symbol $\mathcal{P}_{s}$. The received signal at the relay $\mathrm{R}$ is given by

$$
\boldsymbol{y}_{\mathrm{R}}=h_{1} \boldsymbol{s}_{1}+h_{2} \boldsymbol{x}_{1}+\boldsymbol{n}_{\mathrm{R}}
$$

where $s_{1}=\left[\begin{array}{ll}s_{1} & s_{2}\end{array}\right]$ and $x_{1}=\left[\begin{array}{ll}x_{1} & x_{2}\end{array}\right]$ are the two first rows of the Alamouti codes generated by $S_{1}$ and $S_{2}$, respectively. The channel coefficients for the link from $S_{1}$ and $\mathrm{S}_{2}$ to $\mathrm{R}$, respectively denoted as $h_{1}$ and $h_{2}$, follow a Rayleigh distribution, i.e., $h_{1} \sim \mathcal{C N}\left(0, \Omega_{1}\right)$ and $h_{2} \sim \mathcal{C N}\left(0, \Omega_{2}\right)$. The vector $n_{\mathrm{R}}$ is the complex additive white Gaussian noise (AWGN) with zero mean and variance $N_{0}$.

In the second phase, $\mathrm{R}$ amplifies the received signal, i.e., $\boldsymbol{y}_{\mathrm{R}}$, with the same power constraint as in the first phase and forwards to $S_{2}$ while $S_{1}$ sends the second row of the Alamouti code to $S_{2}$. The received signal at the source $S_{2}, \boldsymbol{y}_{2}$, is written as

$$
\boldsymbol{y}_{2}=h_{2} G \boldsymbol{y}_{\mathrm{R}}+h_{0} \boldsymbol{s}_{2}+\boldsymbol{n}_{2}
$$

where $G$ is the scalar amplifying gain at the relay, $h_{0} \sim$ $\mathcal{C N}\left(0, \Omega_{0}\right)$ is the channel gain for the link between $S_{1}$ and $S_{2}$, $\boldsymbol{s}_{2}=\left[\begin{array}{ll}-s_{2}^{*} & s_{1}^{*}\end{array}\right]$ is the second row of the Alamouti code, and $\boldsymbol{n}_{2}$ is the AWGN vector with zero mean and variance $N_{0}$.
Similarly, in the third phase, R transmit $G \boldsymbol{y}_{\mathrm{R}}$ whereas $\mathrm{S}_{2}$ sends the second row of the Alamouti code to $S_{1}$. The received signal at the source $S_{1}, \boldsymbol{y}_{1}$, is shown as

$$
\boldsymbol{y}_{1}=h_{1} G \boldsymbol{y}_{\mathrm{R}}+h_{0} \boldsymbol{x}_{2}+\boldsymbol{n}_{1}
$$

where $\boldsymbol{x}_{2}=\left[\begin{array}{ll}-x_{2}^{*} & x_{1}^{*}\end{array}\right]$ is the second row of Alamouti code and $\boldsymbol{n}_{1}$ is the AWGN vector with zero mean and variance $N_{0}$.

The amplifying gain $G$ is determined to satisfy the average power constraint between the relay and source, i.e., $\mathrm{R}$ consumes the same amount of power as each source. In this paper, we assume channel state information (CSI)-assisted AF relay, i.e., the relay perfectly knows instantaneous values of the channel gains $h_{1}$ and $h_{2}$. Taking these information into account, we have

$$
\mathbb{E}\left\{\left\|G \boldsymbol{y}_{\mathrm{R}}\right\|_{\mathrm{F}}^{2}\right\}=\mathbb{E}\left\{\left\|\boldsymbol{s}_{i}\right\|_{\mathrm{F}}^{2}\right\}=\mathbb{E}\left\{\left\|\boldsymbol{x}_{i}\right\|_{\mathrm{F}}^{2}\right\}
$$

yielding

$$
G^{2}=\left(\left|h_{1}\right|^{2}+\left|h_{2}\right|^{2}+\frac{1}{\bar{\gamma}}\right)^{-1}
$$

where $\bar{\gamma}=\mathcal{P}_{s} / N_{0}$ is the average signal-to-noise ratio (SNR). With sufficient large SNR, the amplifying gain can be tightly approximated as

$$
G^{2} \approx\left(\left|h_{1}\right|^{2}+\left|h_{2}\right|^{2}\right)^{-1}
$$

It is assumed that the two sources have perfect knowledge of the corresponding channel coefficients to fully cancel selfinterference. Hence, the received signal at $S_{1}$ and $S_{2}$ given in (3) and (2) can readily be formed, respectively, as follows:

$$
\begin{aligned}
& \boldsymbol{y}_{1}=\boldsymbol{h} \boldsymbol{X}+\boldsymbol{z}_{1} \\
& \boldsymbol{y}_{2}=\boldsymbol{h} \boldsymbol{S}+z_{2}
\end{aligned}
$$

where $\boldsymbol{h}=\left[\begin{array}{ll}G h_{1} h_{2} & h_{0}\end{array}\right], \boldsymbol{z}_{1}=G h_{1} \boldsymbol{n}_{\mathrm{R}}+\boldsymbol{n}_{1}, \boldsymbol{z}_{2}=$ $G h_{2} \boldsymbol{n}_{\mathrm{R}}+\boldsymbol{n}_{2}, \boldsymbol{S}=\left[\begin{array}{cc}s_{1} & s_{2} \\ -s_{2}^{*} & s_{1}^{*}\end{array}\right]$, and $\boldsymbol{X}=\left[\begin{array}{cc}x_{1} & x_{2} \\ -x_{2}^{*} & x_{1}^{*}\end{array}\right]$. Here, the new noise vector $z_{i}$, with $i \in\{1,2\}$, is the complex Gaussian vector, i.e., $\boldsymbol{z}_{i} \sim \tilde{\mathcal{N}}_{2}\left(\mathbf{0}, N_{0}\left(1+\left|h_{i}\right|^{2} G^{2}\right) \boldsymbol{I}_{2}\right)$. It is important to note that $\boldsymbol{S}$ and $\boldsymbol{X}$ are Alamouti space-time codes constructed at $S_{1}$ and $S_{2}$, respectively.

\section{Average Sum-Rate of Distributed Alamouti \\ Space-Time Codes with Two-Way AF Relaying}

In this section, we investigate the information-theoretic performance of the proposed DASTC with two-way AF relaying presented in Section [I As mentioned above, since the channel model is assumed to be ergodic block fading, we can describe the average sum-rate as follows:

$$
\begin{aligned}
C_{\text {sum }} & =\mathbb{E}\left\{\frac{2}{3} \log _{2} \operatorname{det}\left(\boldsymbol{I}_{2}+\frac{1}{N_{0}\left(1+G^{2}\left|h_{2}\right|^{2}\right)} \boldsymbol{h}^{\dagger} \boldsymbol{R}_{\boldsymbol{s}} \boldsymbol{h}\right)\right. \\
& \left.+\frac{2}{3} \log _{2} \operatorname{det}\left(\boldsymbol{I}_{2}+\frac{1}{N_{0}\left(1+G^{2}\left|h_{1}\right|^{2}\right)} \boldsymbol{h}^{\dagger} \boldsymbol{R}_{\boldsymbol{x}} \boldsymbol{h}\right)\right\}
\end{aligned}
$$

where $\boldsymbol{R}_{\boldsymbol{s}}$ and $\boldsymbol{R}_{\boldsymbol{x}}$ are the covariance matrices of Gaussian codewords and the pre-factor $2 / 3$ accounts for the fact that each source received information consisting of two symbols 
over three time slots. Due to the orthogonality of Alamouti codeword, it is easy to see that $\boldsymbol{R}_{\boldsymbol{s}}=\mathbb{E}\left\{\boldsymbol{S} \boldsymbol{S}^{\dagger}\right\}=2 \mathcal{P}_{s} \boldsymbol{I}_{2}$ and $\boldsymbol{R}_{\boldsymbol{x}}=\mathbb{E}\left\{\boldsymbol{X} \boldsymbol{X}^{\dagger}\right\}=2 \mathcal{P}_{s} \boldsymbol{I}_{2}$. Then we can rewrite (9) as

$$
\begin{aligned}
& C_{\text {sum }}= \\
& \mathbb{E}\left\{\frac { 2 } { 3 } \operatorname { l o g } _ { 2 } \left[\left(1+2 \bar{\gamma} \frac{\left|h_{1}\right|^{2}\left|h_{2}\right|^{2}+\left|h_{0}\right|^{2}\left(\left|h_{1}\right|^{2}+\left|h_{2}\right|^{2}\right)}{\left|h_{1}\right|^{2}+2\left|h_{2}\right|^{2}}\right)\right.\right. \\
& \left.\left.\times\left(1+2 \bar{\gamma} \frac{\left|h_{1}\right|^{2}\left|h_{2}\right|^{2}+\left|h_{0}\right|^{2}\left(\left|h_{1}\right|^{2}+\left|h_{2}\right|^{2}\right)}{\left|h_{2}\right|^{2}+2\left|h_{1}\right|^{2}}\right)\right]\right\}
\end{aligned}
$$

where (10) is established by the fact that $\operatorname{det}(I+A B)=$ $\operatorname{det}(\boldsymbol{I}+\boldsymbol{B} \boldsymbol{A})$. For notational simplicity, let us denote $\gamma_{i}=$ $2 \bar{\gamma}\left|h_{i}\right|^{2}$ with $i=0,1,2$. Moreover, in the high SNR regime, i.e., $\bar{\gamma}$ is sufficient large, the average sum-rate $C_{\text {sum }}$ given in (10), after some manipulations, can be tightly approximated as

$$
\begin{aligned}
\frac{3 \ln 2}{2} C_{\text {sum }} & \approx \underbrace{\mathbb{E}\left\{\ln \left(\frac{\gamma_{1} \gamma_{2}}{\gamma_{1}+2 \gamma_{2}}\right)\right\}}_{I_{1}}+\underbrace{\mathbb{E}\left\{\ln \left(\frac{\gamma_{1} \gamma_{2}}{\gamma_{2}+2 \gamma_{1}}\right)\right\}}_{I_{2}} \\
& +2 \underbrace{\mathbb{E}\left\{\ln \left(1+\frac{\gamma_{0}\left(\gamma_{1}+\gamma_{2}\right)}{\gamma_{1} \gamma_{2}}\right)\right\}}_{I_{3}}
\end{aligned}
$$

In order to calculate (11), we first start our derivation by considering the similarity between $I_{1}$ and $I_{2}$ in (11). To evaluate the integral $I_{1}$ we utilize the probability density function (PDF) of $Z$ derived in Lemma1 1 given in the Appendix. From (25), we have

$$
I_{1}=\sum_{u=1}^{2} \sum_{v=0}^{1} \int_{0}^{\infty} \mathcal{A}_{v} \frac{\ln z}{1+z} z^{u} \exp \left(-\beta_{1} z\right) \mathcal{K}_{v}(\alpha z) d z
$$

where

$$
\begin{array}{rlrl}
\alpha & =2 \sqrt{\frac{2}{\bar{\gamma}_{1} \bar{\gamma}_{2}}}, & & \beta_{1}=\frac{1}{\bar{\gamma}_{2}}+\frac{2}{\bar{\gamma}_{1}} \\
\mathcal{A}_{0} & =\alpha^{2}, & \mathcal{A}_{1}=\alpha \beta_{1}
\end{array}
$$

To further simplify (12), we will express $\ln z /(1+z)$ and $\exp (-\alpha z) \mathcal{K}_{v}(\alpha z)$ in terms of the Meijer's G-function with the help of [13, eq. (8.4.6.13)] and [13, eq. (6.4.23.3)] as follows:

$$
\begin{aligned}
\frac{\ln z}{1+z} & =-\pi G_{3,3}^{2,2}\left(\begin{array}{c|c}
z & 0,1 / 2 \\
0,0,1 / 2
\end{array}\right) \\
\exp (-\alpha z) \mathcal{K}_{v}(\alpha z) & =\sqrt{\pi} G_{1,2}^{2,0}\left(\begin{array}{l|c}
2 \alpha z & 1 / 2 \\
v,-v
\end{array}\right)
\end{aligned}
$$

where $G_{p, q}^{m, n}(\cdot)$ is the Meijer's G-function [13, eq. (8.2.1.1)]. Moreover, the Meijer's G-function is a special case of the Fox's H-function [13, eq. (8.3.2.21)]

$$
G_{p, q}^{m, n}\left(z \mid \begin{array}{c}
\left(a_{p}\right) \\
\left(b_{q}\right)
\end{array}\right)=H_{p, q}^{m, n}\left(\begin{array}{l}
z \\
\left(a_{p}, 1\right) \\
\left(b_{q}, 1\right)
\end{array}\right)
$$

By combining (13), (14), and (15) with (12), we obtain

$$
\begin{aligned}
I_{1}=- & \sum_{u=1}^{2} \sum_{v=0}^{1} \pi^{3 / 2} \mathcal{A}_{v} \int_{0}^{\infty} z^{u} \exp \left[-\left(\beta_{1}-\alpha\right) z\right] \\
& \times H_{3,3}^{2,2}\left[z \mid \begin{array}{c}
(0,1),(0,1),(1 / 2,1) \\
(0,1),(0,1),(1 / 2,1)
\end{array}\right] \\
& \times H_{1,2}^{2,0}\left[\begin{array}{l|c}
2 \alpha z & (1 / 2,1) \\
(v, 1),(-v, 1)
\end{array}\right] d z
\end{aligned}
$$

Then, the integral $I_{1}$ can be calculated with the help of [14, eq. (2.6.2)] as follows:

$$
\begin{aligned}
& I_{1}=-\pi^{3 / 2} \sum_{u=1}^{2} \sum_{v=0}^{1} \mathcal{A}_{v}\left(\beta_{1}-\alpha\right)^{-u-1} \\
& H_{1,[3: 1], 0,[3: 2]}^{1,2,0,2}\left[\begin{array}{c|c}
\frac{1}{\alpha+\beta_{1}} & (1+u, 1) \\
& (1 / 1,1) ;(0,1),(0,1),(1 / 2,1) \\
\frac{2 \alpha}{\alpha+\beta_{1}} & (0,1),(0,1),\left(\frac{1}{2}, \overline{1}\right) ;(v, 1),(-v, 1)
\end{array}\right]
\end{aligned}
$$

where $H_{E,[A: C], F,[B: D]}^{K, N, N^{\prime}, M, M^{\prime}}$ is the generalized Fox's $H$-function [14, eq. (2.2.1)]. Similarly, we can obtain the closed-form expression for $I_{2}$.

We next evaluate the integral $I_{3}$ by utilizing the PDF of $T$ derived in Lemma 2 given in the Appendix. From (32), we have

$$
\begin{aligned}
I_{3}=\sum_{n=1}^{2} \xi_{n} & \int_{0}^{\infty} \ln (1+t)(t+\zeta)^{-3-n} \\
& \times{ }_{2} F_{1}\left(3+n, n+\frac{1}{2} ; \frac{7}{2} ; \frac{t+\eta}{t+\zeta}\right) d t
\end{aligned}
$$

Then we exchange the variable $\frac{t+\eta}{t+\zeta}=\frac{x}{x+1}$ and assume that the relaying protocol is symmetric, i.e., $\bar{\gamma}_{1}=$ $\bar{\gamma}_{2}$, yielding $\eta=0$. We next express $\ln (1+\zeta x)$ and $(1+x)_{2}^{-a} F_{1}\left(a, b ; c ; \frac{x}{x+1}\right)$ in terms of the Meijer's Gfunction with the help of [13, eq. (8.4.6.5)] and [13, eq. (8.4.49.26)] as follows:

$$
\begin{array}{r}
\ln (1+\zeta x)=G_{2,2}^{1,2}\left(\zeta x \mid \begin{array}{c}
1,1 \\
1,0
\end{array}\right) \\
(1+x)_{2}^{-3-n} F_{1}\left(3+n, n+\frac{1}{2} ; \frac{7}{2} ; \frac{x}{x+1}\right) \\
=G_{2,2}^{1,2}\left(x \mid \begin{array}{c}
-2-n, \frac{1}{2}-n \\
0,-2 n
\end{array}\right)
\end{array}
$$

By combining (19) and (20) with (18), we have

$$
\begin{array}{r}
I_{3}=\sum_{n=1}^{2} \xi_{n} \int_{0}^{\infty} G_{2,2}^{1,2}\left(x \mid \begin{array}{c}
-2-n, \frac{1}{2}-n \\
0,-2 n
\end{array}\right) \\
\times G_{2,2}^{1,2}\left(\begin{array}{l|l}
\zeta x & 1,1 \\
1,0
\end{array}\right) d x
\end{array}
$$


The integral given in (21) can be finalized in the form of Fox's $\mathrm{H}$-function by using [15, eq. (7.811.1)] and [13, eq. (8.3.2.21)] yielding $I_{3}$ as follows:

$$
I_{3}=\sum_{n=1}^{2} \xi_{n} H_{4,4}^{4,2}\left[\zeta \mid \begin{array}{c}
(0,1),(2 n, 1),(1,1),(1,1) \\
(2+n, 1),\left(n-\frac{1}{2}, 1\right),(1,1),(0,1)
\end{array}\right]
$$

By substituting (17) and (22) in (11) and considering the fact that $I_{2}$ can be obtained in a similar form of $I_{1}$, the sum-rate of DASTC with two-way AF relay can be shown as

$$
\begin{aligned}
& C_{\text {sum }} \approx-\frac{2 \pi^{3 / 2}}{3 \ln 2} \sum_{k=1}^{2} \sum_{u=1}^{2} \sum_{v=0}^{1} \mathcal{A}_{v}\left(\beta_{k}-\alpha\right)^{-u-1} \\
& H_{1,[3: 1], 0,[3: 2]}^{1,2,0,2}\left[\begin{array}{c|c}
\frac{1}{\alpha+\beta_{k}} & (1+u, 1) \\
& (1 / 1,1) ;(0,1),(0,1),(1 / 2,1) \\
\frac{2 \alpha}{\alpha+\beta_{k}} & (0,1),(0,1),\left(\frac{1}{2}, 1\right) ;(v, 1),(-v, 1)
\end{array}\right] \\
& +\frac{4}{3 \ln 2} \sum_{n=1}^{2} \xi_{n} H_{4,4}^{4,2}\left[\begin{array}{c}
(0,1),(2 n, 1),(1,1),(1,1) \\
(2+n, 1),\left(n-\frac{1}{2}, 1\right),(1,1),(0,1)
\end{array}\right]
\end{aligned}
$$

\section{Numerical Results}

In this section, we provide the numerical results to verify the proposed two-way DASTC and the correctness of our analysis in two specific examples. The path loss of each link follows an exponential-decay model: if the distance between the two sources is equal to $d$, then $\Omega_{0} \propto d^{-\alpha}$ where an exponent of $\alpha=4$ corresponds to a typical non line-of-sight propagation [7]. Here we assume that the $S_{1} \longrightarrow S_{2}$ link has unit channel mean power, i.e., $\Omega_{0}=1$. In the first example, we consider the symmetric relaying protocol, i.e., $\Omega_{0}=\Omega_{1}=\Omega_{2}=1$. In the second example, we assume that the geometry for locations of three communicating terminals is co-linear where the relay is placed half way between the two sources, i.e., $\Omega_{1}=\Omega_{2}=16$ and $\Omega_{0}=1$.

Fig. 2 and Fig. 3 illustrate the average sum-rate of DASTC in two-way AF relay networks versus average SNR for the two considered examples. As can be observed from these two figures, the analysis is very tight from the middle to high SNR regime. Specifically, from $S N R=10 \mathrm{~dB}$ the analytical and simulation curves perfectly match with each other which verify the tightness of our approximation.

More importantly, the proposed two-way DASTC scheme outperforms the conventional one-way system in terms of the spectral efficiency in the whole considered range of SNR. In particular, at $\mathrm{SNR}=30 \mathrm{~dB}$, the proposed scheme enhances the average sum-rate to $3.8 \mathrm{bps} / \mathrm{Hz}$ in both Example 1 and 2 compared to the conventional one. It is interesting to observe that with a fixed value of $\Omega_{0}$ the gain is unchanged, irrespective of the relay's location.

\section{CONCLUSIONS}

We have proposed a DASTC scheme for two-way AF relay networks that circumvents the loss in spectral efficiency inherently occurred in conventional one-way DASTC system.

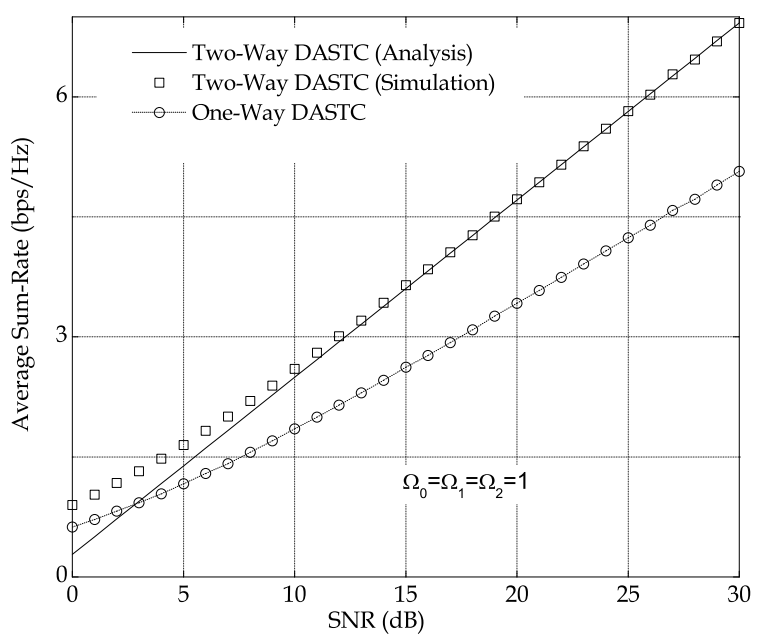

Fig. 2. Average sum-rate of DASTC with symmetric relaying protocol.

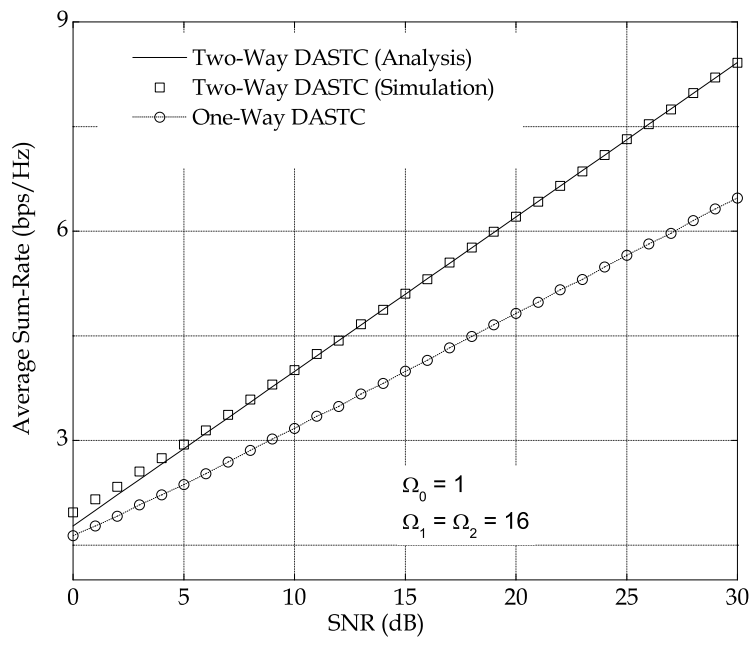

Fig. 3. Average sum-rate of DASTC with co-linear relaying protocol.

We also derive the tight approximation for the average sumrate of the proposed scheme. The closed-form expression for approximated sum-rate is given in the form of Fox's $\mathrm{H}$-function which readily allows us to analyze the spectral efficiency of the proposed scheme. The numerical results provided have validated our analysis.

\section{APPENDIX}

The following lemmas will be helpful in the paper.

Lemma 1: Let $\gamma_{i}$ and $\gamma_{j}$ be statistically independent and not necessarily identically distributed (i.n.i.d.) exponential random variables with hazard rate $\bar{\gamma}_{i}$ and $\bar{\gamma}_{j}$, respectively. Suppose that the ratio of $Z$ takes the form

$$
Z=\frac{\gamma_{i} \gamma_{j}}{\gamma_{i}+2 \gamma_{j}}
$$

Then, we obtain the PDF of random variable $Z$ as

$$
p_{Z}(z)=\alpha z \exp \left(-\beta_{i} z\right)\left[\alpha \mathcal{K}_{0}(\alpha z)+\beta_{i} \mathcal{K}_{1}(\alpha z)\right]
$$

where $\alpha=2 \sqrt{\frac{2}{\bar{\gamma}_{i} \bar{\gamma}_{j}}}$ and $\beta_{i}=\frac{1}{\bar{\gamma}_{j}}+\frac{2}{\bar{\gamma}_{i}}$. 
Proof: Let us rewrite $Z$ in a more tractable form as

$$
Z=(X+Y)^{-1}
$$

where $X=1 / \gamma_{i}$ and $Y=2 / \gamma_{j}$. Then, after some algebraic simplifications together with the help of [15, eq. (3.471.9)], the moment generating function (MGF) of $X$ and $Y$ can be expressed as, respectively

$$
\begin{aligned}
& \Phi_{X}(s)=\mathbb{E}_{X}\{\exp (-s x)\}=2 \sqrt{\frac{s}{\bar{\gamma}_{i}}} \mathcal{K}_{1}\left(2 \sqrt{\frac{s}{\bar{\gamma}_{i}}}\right) \\
& \Phi_{Y}(s)=\mathbb{E}_{Y}\{\exp (-s y)\}=2 \sqrt{\frac{2 s}{\bar{\gamma}_{j}}} \mathcal{K}_{1}\left(2 \sqrt{\frac{2 s}{\bar{\gamma}_{j}}}\right)
\end{aligned}
$$

As can be seen from (26), since $1 / Z$ is the sum of two statistically independent random variables, by using (27) and (28), the MGF of $1 / Z$ can be expressed as

$$
\Phi_{1 / Z}(s)=4 \sqrt{\frac{2}{\bar{\gamma}_{i} \bar{\gamma}_{j}}} s \mathcal{K}_{1}\left(2 \sqrt{\frac{s}{\bar{\gamma}_{i}}}\right) \mathcal{K}_{1}\left(2 \sqrt{\frac{2 s}{\bar{\gamma}_{j}}}\right)
$$

Then, the cumulative distribution function (CDF) of $1 / Z$, $F_{1 / Z}(x)$, can be shown as

$$
F_{1 / Z}(x)=1-\left.\mathcal{L}^{-1}\left\{\Phi_{1 / Z}(s) / s\right\}\right|_{1 / x}
$$

where $\mathcal{L}^{-1}\{$.$\} stands for the inverse Laplace transform. From$ (29) and (30) together with [16, eq. (13.2.20)], we have

$$
F_{Z}(z)=1-\alpha z \exp \left(-\beta_{i} z\right) \mathcal{K}_{1}(\alpha z)
$$

where $\alpha=2 \sqrt{\frac{2}{\bar{\gamma}_{i} \bar{\gamma}_{j}}}$ and $\beta_{i}=\frac{1}{\bar{\gamma}_{j}}+\frac{2}{\bar{\gamma}_{i}}$. By differentiating 311 with respect to $z$ and using the fact that $\frac{d \mathcal{K}_{v}(z)}{d z}=-\mathcal{K}_{v-1}(z)-$ $\frac{v}{z} \mathcal{K}_{v}(z)$ [15, eq. (8.486.12)], we obtain (25) which completes the proof.

Lemma 2: Let $\gamma_{0}, \gamma_{1}$, and $\gamma_{2}$ be the three i.n.i.d. exponential random variables with hazard rates $\bar{\gamma}_{0}, \bar{\gamma}_{1}$, and $\bar{\gamma}_{2}$, respectively, then the PDF of $T=\gamma_{0} \frac{\gamma_{1}+\gamma_{2}}{\gamma_{1} \gamma_{2}}$ is given by

$$
p_{T}(t)=\sum_{n=1}^{2} \xi_{n} \frac{1}{(t+\zeta)^{3+n}} 2 F_{1}\left(3+n, n+\frac{1}{2} ; \frac{7}{2} ; \frac{t+\eta}{t+\zeta}\right)
$$

where

$$
\begin{aligned}
\xi_{n} & =\sqrt{\pi}\left(\frac{4}{\sqrt{\bar{\gamma}_{1} \bar{\gamma}_{2}}}\right)^{n} \frac{\Gamma(3+n) \Gamma(3-n)}{\Gamma(7 / 2)} \varpi_{n} \\
\varpi_{0} & =\frac{4}{\bar{\gamma}_{0} \bar{\gamma}_{1} \bar{\gamma}_{2}}, \quad \varpi_{1}=\frac{2\left(\bar{\gamma}_{1}+\bar{\gamma}_{2}\right)}{\bar{\gamma}_{0}\left(\bar{\gamma}_{1} \bar{\gamma}_{2}\right)^{3 / 2}} \\
\zeta & =\bar{\gamma}_{0}\left(\frac{1}{\bar{\gamma}_{1}}+\frac{1}{\bar{\gamma}_{2}}+\frac{2}{\bar{\gamma}_{1} \bar{\gamma}_{2}}\right), \quad \eta=\bar{\gamma}_{0}\left(\frac{1}{\bar{\gamma}_{1}}+\frac{1}{\bar{\gamma}_{2}}-\frac{2}{\bar{\gamma}_{1} \bar{\gamma}_{2}}\right)
\end{aligned}
$$

and ${ }_{2} F_{1}(a, b ; c ; z)$ is the Gauss hypergeometric function [17, eq. (2.12.1)]. have

Proof: Since $T=\gamma_{0} \frac{\gamma_{1}+\gamma_{2}}{\gamma_{1} \gamma_{2}}$, by defining $W=\frac{\gamma_{1} \gamma_{2}}{\gamma_{1}+\gamma_{2}}$, we

$$
F_{T}(t)=\int_{0}^{\infty}\left[1-\exp \left(-\frac{w t}{\bar{\gamma}_{0}}\right)\right] p_{W}(w) d w
$$

Following similar steps as in Lemma1, we obtain $p_{W}(w)$ as

$$
\begin{aligned}
& p_{W}(w)=\frac{2 w}{\bar{\gamma}_{1} \bar{\gamma}_{2}} \exp \left[-\left(\frac{1}{\bar{\gamma}_{1}}+\frac{1}{\bar{\gamma}_{2}}\right) w\right] \\
& \times\left[2 \mathcal{K}_{0}\left(\frac{2 w}{\sqrt{\bar{\gamma}_{1} \bar{\gamma}_{2}}}\right)+\left(\frac{\bar{\gamma}_{1}+\bar{\gamma}_{2}}{\bar{\gamma}_{1} \bar{\gamma}_{2}}\right) \mathcal{K}_{1}\left(\frac{2 w}{\sqrt{\bar{\gamma}_{1} \bar{\gamma}_{2}}}\right)\right]
\end{aligned}
$$

By substituting (34) in (33) and taking the derivative with respect to $t$, we obtain the PDF of $T$ as

$$
\begin{gathered}
p_{T}(t)=\sum_{n=1}^{2} \varpi_{n} \int_{0}^{\infty} w^{2} \exp \left[-\left(\frac{t}{\bar{\gamma}_{0}}+\frac{1}{\bar{\gamma}_{1}}+\frac{1}{\bar{\gamma}_{2}}\right) w\right] \\
\times \mathcal{K}_{n}\left(\frac{2 w}{\sqrt{\bar{\gamma}_{1} \bar{\gamma}_{2}}}\right) d w
\end{gathered}
$$

It is observed that the integral given in (35) can be simplified by using [13, eq. (6.621.3)] which results in (32). This completes the proof.

\section{REFERENCES}

[1] B. Rankov and A. Wittneben, "Spectral efficient protocols for halfduplex fading relay channels," IEEE J. Sel. Areas Commun., vol. 25, no. 2, pp. 379-389, Feb. 2007.

[2] P. Popovski and H. Yomo, "Wireless network coding by amplify-andforward for bi-directional traffic flows," IEEE Commun. Lett., vol. 11, no. 1, pp. 16-18, Jan. 2007.

[3] T. Unger and A. Klein, "Duplex schemes in multiple antenna two-hop relaying," EURASIP Journal on Advances in Signal Processing, vol. 2008, article ID 128592, 14 pages, 2008. doi:10.1155/2008/128592.

[4] T. Q. Duong, L.-N. Hoang, and V. N. Q. Bao, "On the performance of two-way amplify-and-forward relay networks," IEICE Trans on Commun., vol. Vol.E92-B, no. 12, pp. 3957-3959, Dec. 2009.

[5] R. H. Y. Louie, Y. Li, and B. Vucetic, "Practical physical layer network coding for two-way relay channels: Performance analysis and comparison," IEEE Trans. Wireless Commun., vol. 9, no. 2, pp. 764-777, Feb. 2010.

[6] T. Q. Duong, D.-B. Ha, H.-A. Tran, and N.-S. Vo, "Symbol error probability of distributed-Alamouti scheme in wireless relay networks," in Proc. 67th IEEE Veh. Technol. Conf. Spring, Marina Bay, Singapore, May 2008, pp. 648-652.

[7] Y. Han, S. H. Ting, C. K. Ho, and W. H. Chin, "High rate two-way amplify-and-forward half-duplex relaying with OSTBC," in Proc. 67th IEEE Veh. Technol. Conf. Spring, Singapore, Apr. 2008, pp. 2426-2430.

[8] _ , "Performance bounds for two-way amplify-and-forward relaying," IEEE Trans. Wireless Commun., vol. 8, no. 1, pp. 432-439, Jan. 2009.

[9] M. Eslamifar, W. H. Chin, C. Yuen, and G. Y. Liang, "Performance analysis of two-way multiple-antenna relaying with network coding," in Proc. 70th IEEE Veh. Technol. Conf. Fall, Alaska, U.S.A., Sep. 2009, pp. 1-5.

[10] R. U. Nabar, H. Bölcskei, and F. W. Kneubühler, "Fading relay channels: Performance limits and space-time signal design," IEEE J. Sel. Areas Commun., vol. 22, no. 8, pp. 1099-1109, Aug. 2004.

[11] T. Q. Duong, N.-T. Nguyen, T. Hoang, and V.-K. Nguyen, "Pairwise error probability of distributed space-time coding employing Alamouti scheme in wireless relays networks," Wireless Personal Communications, vol. 51, no. 2, pp. 231-244, Oct. 2009.

[12] S. M. Alamouti, "A simple transmit diversity technique for wireless communications," IEEE J. Sel. Areas Commun., vol. 16, no. 8, pp. 14511458, Oct. 1998.

[13] A. P. Prudnikov, Y. A. Brychkov, and O. I. Marichev, Integrals and Series. New York: Gordon and Breach Science, 1990, vol. 3.

[14] A. M. Mathai and R. K. Saxena, The H-function with Applications in Statistics and Other Disciplines. New York: Wiley, 1978.

[15] I. S. Gradshteyn and I. M. Ryzhik, Table of Integrals, Series, and Products, 6th ed. San Diego, CA: Academic Press, 2000.

[16] G. E. Roberts and H. Kaufman, Table of Laplace Transforms. W. B. Saunders, 1966.

[17] A. Erdelyi, Higher Transcendental Functions. New York: McGrawHill, 1953, vol. 1 . 